\title{
STUART CLOTTING DEFECT. II. GENETIC ASPECTS OF A 'NEW' HEMORRHAGIC STATE
}

\author{
By JOHN B. GRAHAM, EMILY M. BARROW, AND CECIL HOUGIE 1
}

(From the Department of Pathology, University of North Carolina, Chapel Hill, N. C.)

(Submitted for publication August 24, 1956; accepted November 29, 1956)

The literature is ambiguous regarding patients deficient in the clotting factor variously known as "SPCA," "proconvertin," and "Factor VII." Sera from some patients are normal in the thromboplastin generation test; those from others are abnormal. Viper venom corrects the clotting defect in some cases, but fails to do so in others. Despite these contradictions, it has been generally assumed that the terms are synonyms for a single clotting factor with unique characteristics.

In our preceding communication on the patient R. S. (1), previously reported by others as hypoproconvertinemia (2), we pointed out that the assumption of identity must be incorrect, since the plasmas of our patient and the SPCA deficient patient of Alexander, Goldstein, Landwehr, and Cook (3) were mutually corrective, while our patient's plasma failed to correct that of the patient of Crockett, Shotton, Craddock, and Leavell (4). This finding implied the existence of at least two $\mathrm{BaSO}_{4}$ adsorbable clotting factors whose lack prolongs the prothrombin time. We showed that, in contrast to certain other cases in the literature (5-8), the defect in our patient's plasma could not be corrected by Russell viper venom, and that his serum was inactive in the thromboplastin generation test. In this last respect our patient's defect resembled that of some of the previously reported cases $(8-11)$ but differed from certain others $(5,6,12-14)$.

The factor deficient in our patient is being referred to as the Stuart factor after the patient's surname. We wish to emphasize by this nomenclature that only by cross-matching his plasma with that from other similar patients can an identity be definitely established. The properties of Stuart factor and its role in blood coagulation were the subject of our earlier communication (1). In this paper the studies on the relatives of our patient will be reported. Our genetic studies in-

\footnotetext{
1 Present address: Department of Clinical Pathology, University of Virginia, Charlottesville, Virginia.
}

dicate that the Stuart defect is inherited as a highly penetrant, incompletely recessive, autosomal characteristic.

\section{MATERIALS AND METHODS}

Blood for study was obtained from certain members of the family on three separate occasions. On the first field trip, blood was obtained from the proband, his wife, and two of his sons. On the second field trip, blood was obtained during the course of a single day from all the persons shown in Table I. On the third trip, blood was obtained from the proband, his wife, and his daughter. It was established on the first trip that the plasma and serum of the proband's wife did not differ significantly in concentration of Stuart factor from. several, presumably normal, laboratory workers. Henceforth, the wife was used as the control subject, in the belief that a field control was required to cover the manipulations of venipuncture, transportation and storage. On all occasions, blood was obtained by the two syringe, silicone technique and was centrifuged in a portable Servall centrifuge in siliconed centrifuge tubes.

Plasma was pipetted immediately from the centrifuge tubes into storage tubes; prothrombin time was determined at once on a sample, and the remainder was quickly frozen and stored at $-70^{\circ} \mathrm{C}$. The following day, prothrombin times and Stuart factor assays were performed on all samples of plasma in our own laboratory.

Serum was obtained from the members of the family at the same venipuncture as plasma. Whole blood was placed in clean, non-siliconed centrifuge tubes. Tubes were stoppered and the blood allowed to clot, precautions being taken to prevent hemolysis. The clotted blood remained at automobile and room temperature for 18 to 21 hours before centrifugation. The serum was expressed by centrifugation in our own laboratory the next day and tested for residual prothrombin and thrombin. When a serum sample contained detectable prothrombin or thrombin it was placed in a $28^{\circ} \mathrm{C}$ waterbath until the clotting time with thromboplastin plus fibrinogen was greater than 300 seconds, and it did not clot fibrinogen in 10 minutes. This additional incubation was required in only a few cases, notably the proband's. All samples of serum were frozen and stored at $-20^{\circ} \mathrm{C}$, including the control serum from the proband's wife. All thromboplastin generation tests were performed on the second and third day after obtaining the serum. The same $\mathrm{Al}(\mathrm{OH})_{3}$-adsorbed normal plasma and 0.03 per cent 
TABLE I

The plasma and serum Stuart factor levels of fourteen members of the Stuart kindred

\begin{tabular}{clcccc}
\hline \hline & & \multicolumn{2}{c}{ Per cent of Stuart factor } & & \\
\cline { 3 - 5 } Number & 4rothrombin & $\begin{array}{c}\text { Thpln } \\
\text { time } \\
\text { method }\end{array}$ & $\begin{array}{c}\text { Reration } \\
\text { method }\end{array}$ & Average & Probable genotype \\
\hline IV-28 & Proband & $0^{*}$ & $0^{*}$ & 0 & Homozygous abnormal \\
III-11 & Mother & 47 & 40 & 44 & Heterozygous \\
III-23 & Paternal uncle & 44 & 45 & 44 & Heterozygous \\
III-25 & Paternal aunt & 52 & 37 & 44 & Heterozygous \\
V-37 & Son & 32 & 20 & 26 & Heterozygous \\
V-38 & Son & 32 & 22 & 27 & Heterozygous \\
V-39 & Son & 11 & 31 & 21 & Heterozygous \\
V-40 & Son & 32 & 26 & 29 & Heterozygous \\
V-41 & Daughter & 44 & 60 & 52 & Heterozygous \\
III-3 & Maternal aunt & 100 & 100 & 100 & Homozygous normal \\
III-5 & Maternal uncle & 93 & 80 & 86 & Homozygous normal \\
III-21 & Paternal uncle & 100 & 100 & 100 & Homozygous normal \\
IV-7 & Aunt by marriage & 100 & 100 & 100 & Homozygous normal \\
IV-26 & Wife & 100 & 100 & 100 & Homozygous normal \\
\hline
\end{tabular}

* One to three per cent according to Lewis, Fresh, and Ferguson (2).

cephalin suspension (in lieu of platelets) were used in all generation tests.

\section{Stwart factor assays}

Plasma method. The assay procedure was based on a comparison of the correction by control and test plasma of the proband's prolonged prothrombin time. The plasma of the proband (prothrombin time 60 to $85 \mathrm{sec}$ onds) was the substrate for the test. Normal plasma (prothrombin time 12 to 14 seconds) was diluted with the patient's plasma 1:2 through 1:16. Prothrombin time (15) was determined on the various mixtures in triplicate and the values at each dilution were averaged. A calibration curve was constructed by plotting prothrombin time against concentration of the Stuart factor, i.e., the percentage of normal plasma in each mixture, the 1:2 dilution representing 100 per cent.

An unknown plasma was assayed after being diluted $1: 2,1: 4$, and 1:8 with patient's plasma. Prothrombin times were performed on each dilution in triplicate and averaged. Per cent Stuart factor was interpolated from the calibration curve for each dilution of the unknown plasma. The percentage value from the 1:4 dilution was doubled, the value from the $1: 8$ dilution quadrupled, and both were averaged with the value from the $1: 2$ dilution. The final average value for each plasma is shown in Table I.

The arbitrary 100 per cent value in this test is actually equivalent to 50 per cent Stuart factor. An unknown plasma with 50 per cent Stuart factor is, therefore, one which diluted 1:2 with Stuart's plasma, has a clotting time equal to that of the normal control diluted $1: 4$. (A typical dilution curve on normal plasma, November 27 , 1955 , was as follows: $1: 2(100 \%)-14.4$ secs., $1: 4$ (50\%)-16.5 secs., $1: 8(25 \%)-19.6$ secs., $1: 16(12.5 \%)-$ 24 secs.)

Serum method. The Stuart factor content of an unknown serum was determined in the thromboplastin gen- eration test (16) after it had been established that the proband's serum was only about as effective as saline in this test (1). Normal serum was diluted in a serial twofold fashion 1:2 through 1:8 with proband's serum prior to diluting 1:10 with normal saline for use as the serum source in the generation test. The calibration curve was constructed by plotting the minimum clotting time at each dilution (at whatever incubation time) against Stuart factor concentration, i.e., the per cent normal serum in the mixture.

Unknown sera were assayed by diluting $1: 2,1: 4$, and $1: 8$ with patient's serum then $1: 10$ with saline and determining the minimum clotting time in the thromboplastin generation test (at whatever incubation time). Per cent Stuart factor in each dilution of each unknown was determined by interpolation from the calibration curve. The percentage Stuart factor obtained at the 1:4 dilution was multiplied by 2 and that at $1: 8$ by 4 . All three percentage values were averaged and are the averages shown in Table $\mathrm{I}$.

In this assay the $1: 2$ dilution of normal serum with patient's serum was arbitrarily designated 100 per cent on the calibration curve. A value of 50 per cent or less for an unknown serum implies that it had the same or less activity than normal plasma carried one dilution step further. (A typical dilution curve of control serum with patient's serum on November 29,1955 , showed the following minimum clotting times: $1: 2(100 \%)-14.5$ secs., $1: 4$ (50\%)-17 secs., $1: 8(25 \%)-22$ secs.)

\section{PEDIGREE}

The proband belongs to a large kindred living in the Blue Ridge mountains of the northwestern corner of North Carolina and nearby southwestern Virginia. Information was obtained on $164 \mathrm{mem}$ bers of the family; approximately 100 still reside 
within a 30-mile radius of West Jefferson, North Carolina.

The pedigree is shown in Figure 1. The proband (IV-28), a moderately severe bleeder and symptomatic since birth, had not required transfusions until December, 1955, at age 36. His relatives were quite dogmatic that only he should be considered a bleeder. Closer questioning of his wife, however, disclosed that one of his sons (V-39) seemed to have unusually frequent nosebleeds, and that the buttocks of his daughter (V-41) often showed persistent bruises after disciplinary spanking. No history of even a mild bleeding tendency could be elicited from other members of the kindred.

The proband works as a tenant farmer, farm laborer, and lay preacher in the periods between hemorrhagic crises, never dangerous until the latest one. Exacerbations have been of such frequency and irregularity that he has been unable to retain regular employment and has found it difficult to support his family. His chief symptoms have been excessive bleeding from small cuts, occasional hemarthoses and persistent anemia.

It can be seen in Figure 1 that our proband was born of a consanguineous union. His mother (III-11) and father (III-12) were related to each other as aunt and nephew, a not unknown mating type 40 years ago in this isolated mountain area. It should be noted also that there is another but less close consanguineous mating in the pedigree (III-5 to IV-7), in which the abnormal genes were probably absent as judged by assay procedures and 13 apparently normal children.

We assayed for the Stuart factor both plasma and serum from the 14 persons needed to establish a genetic hypothesis. Those available included the proband's mother, children and wife, two of the mother's siblings, and three siblings of his deceased father. The results are shown in Table I. It can be seen that the levels of Stuart factor found with the two procedures agree fairly well. If the values from the two procedures are averaged they fall clearly into three distinct groups. There is an intermediate class between the very low level of the proband ( 1 to 3 per cent according to Lewis and associates [2]) and the normal persons, such as his wife. The mean value for Stuart factor in this intermediate group is 36 per cent with a standard deviation of 9.6 per cent. It is logical, therefore, to hypothesize that the proband is homozygous for an abnormal autosomal gene, that the intermediate class is heterozygous, and that the persons with normal levels are homozygous normal.

This hypothesis can be tested for internal consistency by analyzing the distribution of the heterozygous and normal individuals.

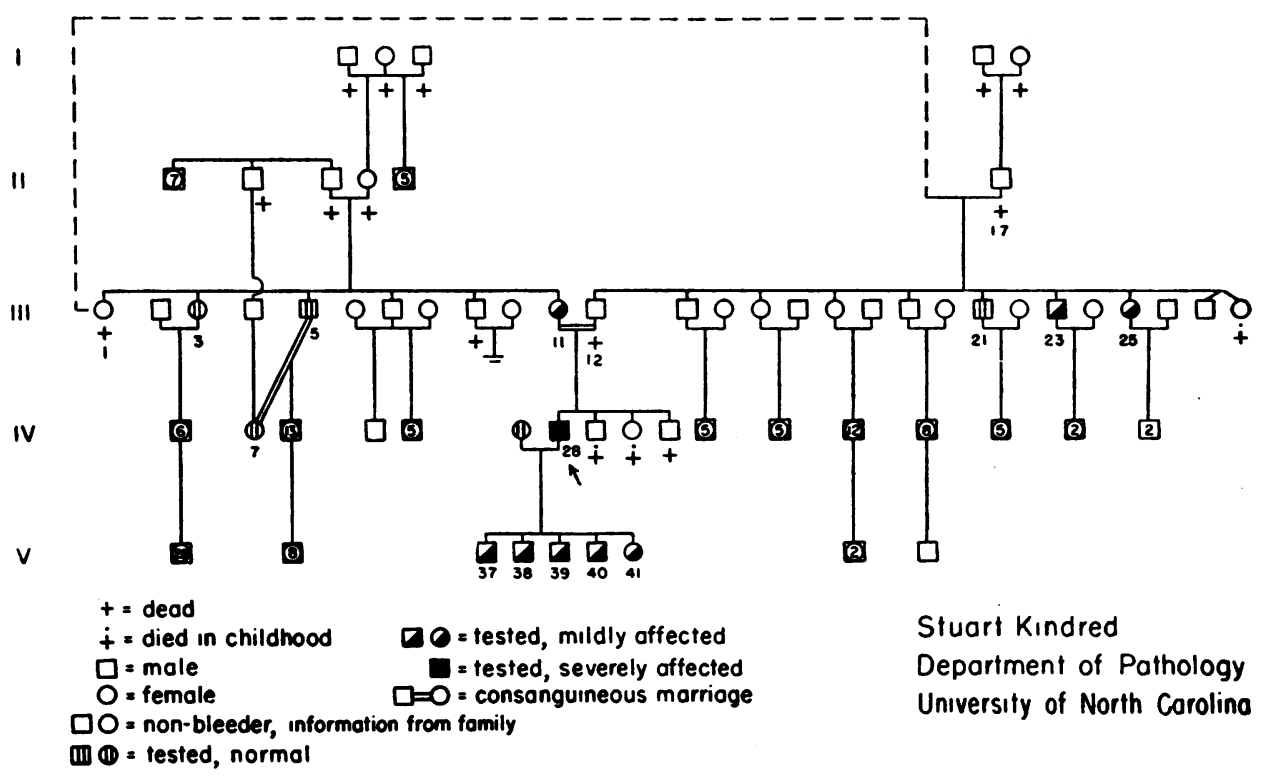

Fig. 1. The Stuart Kindred 
1. The mother of the proband, all his children and two siblings of his (deceased) father are heterozygous as classified by our tests.

2. Both father and mother of the proband have normal siblings.

3. The proband's wife is normal, and there are no homozygous children.

4. The heterozygous siblings of the proband's father, who did not mate consanguineously, do not have clinically affected children.

All these facts are consistent with the hypothesis that the proband is homozygous for an incompletely recessive autosomal gene (st/st), his mother, two of his father's siblings and all his children are heterozygous ( $S t / s t)$, and his maternal aunt, maternal uncle, paternal uncle, aunt by marriage and wife are homozygous normal $(S t / S t)$.

Another hypothesis which might fit these data is that an autosomal gene for the Stuart defect is completely expressed in some individuals in the heterozygous condition (the proband) but very slightly expressed in others (his mother and children). This can be rejected by observing that all five of the proband's children would be required to have received the abnormal gene from their father. If his wife, genetically unrelated and normal by test, were in fact normal (a reasonable assumption), the probability that every child of a heterozygote also would be heterozygous is 1 in 32 or between 3 per cent and 4 per cent, a sufficiently low probability for rejection.

A sex linkage hypothesis can be excluded by noting that the mother and all the children of the proband, four of whom are males, have a mild form of the condition which is severe in him, and that his wife is normal. It is interesting in this connection to note the family history of another case of Stuart factor deficiency, H. H., previously reported as a case of congenital hypoprothrombinemia by Crockett and associates (4) and shown to have Stuart factor deficiency in our earlier publication (1). This second patient is female and her father, mother, and sister appeared normal clinically.

Our hypothesis implies that in the Stuart kindred the sisters, III-1 and III-11, were probably heterozygous at an autosomal locus and that the man, now deceased, who was III-1's son and III-11's husband was also heterozygous. The consanguineous mating of III-11 and III-12 produced at least one homozygote, the proband (IV28). No conclusions can be drawn about the proband's siblings since two died in infancy and the third died of a cerebral vascular accident which might have resulted either from hemorrhage or thrombosis.

Under our hypothesis, one-half of the members of generation III and all of the proband's children should be heterozygous. Reference to Figure 1 will show that this prediction is borne out precisely. Six members of the third generation and all the children were tested. Three of the six adults were clearly heterozygous as were all the children. These facts mean that, assuming our hypothesis to be correct, the abnormal gene is not only incompletely recessive but is also highly penetrant.

Finally, it should be noted that the persons with intermediate levels of the Stuart factor and scored as heterozygous, had $1 \frac{1}{2}$ to 3 -second prolongations in the classical prothrombin time test and minimum clotting times in the routine thromboplastin generation test (serum phase) 2 to 3 seconds longer than control sera.

\section{DISCUSSION}

The data outlined above establish beyond reasonable doubt that Stuart factor deficiency is inherited in this pedigree in an incompletely recessive autosomal fashion, expressed in its most severe form in the homozygote. The homozygote has only a moderately severe hemorrhagic diathesis as was pointed out earlier by Lewis, Fresh, and Ferguson (2). The heterozygotes consider themselves normal, but close questioning discloses that some have a mild tendency to bleed excessively. It is of interest that heterozygosity for this mutation can be detected by appropriate tests. From the theoretical standpoint this finding illustrates again the axiom that every gene has an effect and implies that gene frequency studies based on heterozygote counts are possible. The data of Brink and Kingsley $(17,18)$ and Lewis and Ferguson (19) showing similar effects in 
persons heterozygous for Factor $\mathrm{V}$ deficiency have, perhaps, similar implications.

From the practical standpoint, our data (and the data on Factor V) imply, as Quick has frequently reiterated (see Ref. 20), that a $1 \frac{1}{2}$ to 3 -second prolongation of the prothrombin time should not be shrugged off automatically as a "vagary of the one-stage" provided that it occurs consistently and that the control is reproducible from day-to-day; we would add that a similar occurrence with the thromboplastin generation test may have a similar significance. The observer may be dealing in either instance with an individual heterozygous for an abnormal gene affecting one or another of the prothrombin accessory factors. The obvious conclusion is that all such plasmas should be assayed specifically for the possible deficiencies.

The fact that the heterozygotes in the Stuart kindred did not consider themselves symptomatic does not imply that this is always true. We have recently studied another kindred reputed to be "female bleeders." We discovered that roughly half the females examined had 1 to 3 -second prolongations of plasma prothrombin time and 40 to 60 per cent Stuart factor levels as measured by the assays used in the present communication. These women were known to be bad operative risks by their physicians and to be persistently anemic, presumably because of menorrhagia, since their anemia improved during pregnancy and after menopause. The frequency of the mild disorder in the family and the intermediate Stuart factor levels led us to conclude that the patients were heterozygous.

If the three patients of de Vries, Kettenborg, and van der Pol (9) with abnormal thromboplastin generation tests had Stuart factor deficiency, they were probably heterozygous also, judging from their prothrombin times. It is of interest that all three of these patients were males. Their presenting symptoms, gastrointestinal bleeding, are not surprising in view of the well-known excess $(4: 1)$ of gastro-duodenal ulcer in males. Nevertheless, because of menstruation in women and the increased frequency of operations, it seems likely that more women than men heterozygous for the autosomally transmitted clotting dyscrasias will be referred to the laboratory as doubtful bleeders.

One member of the Stuart kindred, III-5, created a classification problem. However, he was scored as normal since his Stuart factor level ( 86 per cent) was more than 3 standard deviations greater than the mean of the indubitable heterozygotes. This conclusion could have been tested further by examining his 13 children, as half of them would be expected to be heterozygous under our hypothesis if the father were in fact heterozygous. These studies were not carried out, primarily because whether or not he is heterozygous does not affect the main hypothesis. Also since he lives 200 miles from our laboratory in a remote mountain area, testing all his children in the field constitutes a separate enterprise. However, study of his descendants is intended eventually, since it is of more than passing interest to discover whether heterozygosity for the Stuart defect can be present in a person with a normal level of Stuart factor. We have also not yet attempted to study the turnover rate of the Stuart factor in the proband, or the steady-state relationships in the heterozygotes. It is anticipated that these studies will be performed in the future.

A rough calculation of the frequency of the abnormal Stuart gene will be made, because it emphasizes our point about consistently prolonged prothrombin times. We are aware of only one living homozygote for Stuart factor deficiency in North Carolina in a population of somewhat more than $4,000,000$, a frequency of about .00000025 . However, Lewis, Fresh, and Ferguson (2) studied another, now deceased, and Crockett and associates (4) studied an identical patient in Virginia. If this frequency figure is quadrupled to compensate for incomplete ascertainment, it should give a probable upper limit of the frequency (.000001). Then if the Hardy-Weinberg conditions are assumed to apply, the upper limit of the gene frequency can be estimated efficiently as the square root of the frequency of the homozygous class or

$$
q=\sqrt{.000001}=.001
$$

Then the frequency of the normal allele can be obtained as $p=(1-q)$ or $(1-.001)=.999$ and the 
frequency of heterozygotes as

$$
2 \mathrm{pq}=2(.999)(.001)=.0019
$$

or about $2 / 1,000$. This surprisingly high heterozygote frequency implies that if one surveyed a random population of North Carolinians by the prothrombin time test he might encounter as many as two persons in each thousand with prothrombin time consistently prolonged $1 \frac{1}{2}$ to 3 seconds where the prolongation resulted from heterozygosity for Stuart factor deficiency.

Finally, the demonstration that the abnormal autosomal gene for Stuart factor deficiency is incompletely recessive implies that the normal allele is incompletely dominant. This means biochemically either that a single normal allele at the Stuart locus does not furnish enough enzymatic activity or substrate at some point in the synthetic metabolic pathway to maintain the level of the factor at that of "wild type," or that the mutant gene inhibits some step (21). The normal allele at this locus is therefore, in its overall effect, like that for Factor $\mathrm{V}$ in some pedigrees $(18,19)$, some pedigrees of Christmas disease $(22,23)$, and some pedigrees of mild hemophilia (24), and unlike the allele for classic hemophilia $(22,23$, 25).

\section{SUMMARY}

1. Stuart factor deficiency, recently segregated from the heterogeneous group of hemorrhagic states known variously as SPCA, proconvertin or Factor VII deficiency, has been studied in a large North Carolina kindred and shown to be inherited as a highly penetrant but incompletely recessive autosomal characteristic.

2. The heterozygotes have been found to be only mildly affected or normal clinically, but to have $1 \frac{1}{2}$ to 3 -second prolongations of the prothrombin time and thromboplastin generation tests. These effects are, presumably, due to the reduction in the level of this factor to a mean of 36 per cent in the range 20 to 52 per cent.

3. It is pointed out that carrier detection may be possible in many instances with fairly simple tests.

4. It is emphasized that heterozygotes for the various hemorrhagic states, especially females, may be encountered as patients with abnormal operative bleeding, persistent gastro-intestinal bleeding or menorrhagia with persistent anemia. Such symptoms, in the absence of clean-cut laboratory evidence, however, should not be assumed to represent heterozygosity.

\section{REFERENCES}

1. Hougie, C., Barrow, E. M., and Graham, J. B., Stuart clotting defect. I. Segregation of an hereditary hemorrhagic state from the heterogeneous group heretofore called "stable factor" (SPCA, proconvertin, Factor VII) deficiency. J. Clin. Invest., 1957, 36, 485.

2. Lewis, J. H., Fresh, J. W., and Ferguson, J. H., Congenital hypoproconvertinemia. Proc. Soc. Exper. Biol. \& Med., 1953, 84, 651.

3. Alexander, B., Goldstein, R., Landwehr, G., and Cook, C. D., Congenital SPCA deficiency: a hitherto unrecognized coagulation defect with hemorrhage rectified by serum and serum fractions. J. Clin. Invest., 1951, 30, 596.

4. Crockett, C. L., Shotton, D., Craddock, C. G., and Leavell, B. S., Hypoprothrombinemia: studies of a case of the idiopathic type and the effect of serum administration. Blood, 1949, 4, 1298.

5. Jenkins, J. S., Haemorrhagic diathesis due to deficiency of factor VII. J. Clin. Path., 1954, 7, 29.

6. Hicks, N. D., A coagulation disorder due to a factor VII-like defect. M. J. Australia, 1955, 2, 331.

7. Hjort, P., Rapaport, S. I., and Owren, P. A., A simple specific one-stage prothrombin assay using Russell's viper venom in cephalin suspension. J. Lab. \& Clin. Med., 1955, 46, 89.

8. Telfer, T. P., Denson, K. W., and Wright, D. R., A 'new' coagulation defect. Brit. J. Haemat., 1956, 2, 308.

9. de Vries, S. I., Kettenborg, H. K., and van der Pol, E. T., Haemorrhagic diathesis due to a deficiency of Factor VII (Hypoproconvertinaemia). Acta haemat., 1955, 14, 43.

10. Stefanovic, S., Milosavljevic, A., and Stefanovic, R., Deux cas d'hypoconvertinemie congenitale. Sang, 1955, 26, 315.

11. Newcomb, T., Matter, M., Conroy, L., DeMarsh, Q. B., and Finch, C. A., Congenital hemorrhagic diathesis of the prothrombin complex. Am. J. Med., 1956, 20, 798.

12. Alexander, B., 1956, Personal communication.

13. Koch, Fr., Schultze, H. E., Schwick, G., and Beller, F. K., Beobachtungen bei angeborenem Faktor VII-Mangel (Hypokonvertinämie). Ztschr. f. Kinderh., 1955, 76, 208.

14. Jürgens, J., Kongenitaler Factor VII (SPCA)Mangel als Ursache einer hämophilieartigen 
hämorrhagischen Diathese. Acta haemat., 1956, $16,181$.

15. Quick, A. J., The clinical application of the hippuric acid and the prothrombin tests. Am. J. Clin. Path., 1940, 10, 222.

16. Biggs, R., and Douglas, A. S., The thromboplastin generation test. J. Clin. Path., 1953, 6, 23.

17. Brink, A. J., and Kingsley, C. S., A familial disorder of blood coagulation due to deficiency of the labile factor. Quart. J. Med., 1952, 21, 19.

18. Kingsley, C. S., Familial factor V deficiency : the pattern of heredity. Quart. J. Med., 1954, 23, 323.

19. Lewis, J. H., and Ferguson, J. H., Hypoproaccelerinemia. Blood, 1955, 10, 351.

20. Quick, A. J., Pisciotta, A. V., and Hussey, C. V., Congenital hypoprothrombinemic states. Arch. Int. Med., 1955, 95, 2.
21. Haldane, J. B. S., The Biochemistry of Genetics, London, Allen and Unwin Ltd., 1954, pp. 45, 76.

22. Ramot, B., Angelopoulos, B., and Singer, K., Variable manifestations of plasma thromboplastin component deficiency. J. Lab. \& Clin. Med., 1955, 46, 80.

23. Graham, J. B., Biochemical genetics of blood coagulation. Am. J. Human Genet., 1956, 8, 63.

24. Graham, J. B., McLendon, W. W., and Brinkhous, K. M., Mild hemophilia: an allelic form of the disease. Am. J. M. Sc., 1953, 225, 46.

25. Graham, J. B., Collins, D. L., Jr., Godwin, I. D., and Brinkhous, K. M., Assay of plasma antihemophilic activity in normal, heterozygous (hemophilia) and prothrombinopenic dogs. Proc. Soc. Exper. Biol. \& Med., 1951, 77, 294. 